\title{
IMPROVING RELIABILITY OF JUDGMENTAL FORECASTS
}

Stewart, T. R. (2001). Improving reliability of judgmental forecasts. In J. S. Armstrong (Ed.), Principles of Forecasting: A Handbook for Researchers and Practitioners (pp. 81-106): Kluwer Academic Publishers.

Thomas R. Stewart

Center for Policy Research,

Nelson A. Rockefeller College of Public Affairs and Policy,

University at Albany, State University of New York

\section{ABSTRACT}

All judgmental forecasts will be affected by the inherent unreliability, or inconsistency, of the judgment process. Psychologists have studied this problem extensively, but forecasters rarely address it. Researchers and theorists describe two types of unreliability that can reduce the accuracy of judgmental forecasts: (1) unreliability of information acquisition, and (2) unreliability of information processing. Studies indicate that judgments are less reliable when the task is more complex; when the environment is more uncertain; when the acquisition of information relies on perception, pattern recognition, or memory; and when people use intuition instead of analysis. Five principles can improve reliability in judgmental forecasting:

1. Organize and present information in a form that clearly emphasizes relevant information.

2. Limit the amount of information used in judgmental forecasting. Use a small number of really important cues.

3. Use mechanical methods to process information.

4. Combine several forecasts.

5. Require justification of forecasts.

Keywords: Accuracy, combining forecasts, error, information acquisition, information processing, psychometrics, reliability.

People are not consistent. Imperfect reliability (sometimes called "inconsistency") is observed in nearly all human behavior. Observe a person on separate occasions that are identical in every important respect, and you will observe different behavior on each occasion. If a person takes the same test on two different occasions, the two test scores will differ. If 
a person judges the loudness of a sound one day and then judges the same sound the next day, the judgments will usually differ. If a forecaster made a judgmental forecast and then could be somehow transported back in time to repeat the same forecast under identical conditions, she would almost certainly make a different forecast.

In short, unreliability is a source of error in judgmental forecasting. In the long run, it can only reduce the accuracy of forecasts. Lack of reliability or consistency has nothing to do with potentially beneficial behavioral changes over time, such as changes due to learning, obtaining new information, or adapting to new circumstances. Unreliability is simply error introduced into the forecast by the natural inconsistency of the human judgment process.

If human judgment is so important in forecasting, and unreliability is a pervasive and well known (at least to psychologists) source of error in judgment, then why isn't improving reliability a major concern of those who produce and use forecasts? I don't know. One possible reason is that reliability is difficult or impossible to measure directly outside the laboratory. As a result, although we can argue persuasively that a problem exists, it is difficult to demonstrate its importance in operational settings. Another reason is that few psychologists have attempted to explain the practical implications of unreliability. A third is that practitioners may accept inconsistency as an inevitable cost of exercising judgment, or perhaps even mistakenly view it as a benefit. Finally, for all of the above reasons, it is difficult to cite compelling anecdotes about major errors that could be traced to unreliability. The editor of this book asked me to do just that, and I failed, because there are none. I'm confident that errors occur because of unreliability, but they are impossible to detect in a one-time decision (such as the decision to launch the Challenger space shuttle). Separating unreliability from other sources of error requires detailed study of a kind that is rarely done.

Nevertheless, most people do have an intuitive understanding that they are unreliable (although not of how unreliable they are). One of the most common comments made by subjects in my research (who are generally experts being asked to make judgments or forecasts) is "Oh, you are going to find out how inconsistent I am." Furthermore, many of the methods for improving forecasts discussed in this book address reliability, though often implicitly and indirectly. There are many benefits to be gained, however, from explicitly addressing reliability.

\section{THE PROBLEM: IMPERFECT RELIABILITY OF JUDGMENT}

Before discussing the research on reliability of judgment and the forecasting principles that can be derived from it, I need to get some formal definitions and theory out of the way. In the next section, I define reliability as it applies to forecasting and introduce such terms as true score, error, systematic variance, and unsystematic variance. Then, since reliability is such an abstract concept, I have to talk about how it is measured or estimated. Then I introduce the lens-model equation, which quantifies the relation between accuracy and reliability, and an expanded lens model, which shows that there are two types of reliability to worry about. Finally, I will summarize the relevant research on (1) reliability of judgment, (2) reliability of information acquisition, and (3) the implications of analytic and intuitive cognitive processes for reliability. 


\section{Formal Definition of Reliability and its Relation to Error}

Reliability is an important concept in psychological measurement and is extensively discussed in standard psychometrics texts, such as Nunnally (1978). Any test score is assumed to be a sum of a "true" score plus error. Reliability is the square of the correlation between obtained test scores and the underlying true scores. Since the true scores are never known, reliability is typically estimated by correlating the scores on two equivalent tests.

For our purposes, a forecast is analogous to a test score and the true score is the reliable, repeatable component of the forecast. The error cornponent is just random error. When discussing reliability of judgment and forecasts, we do not normally talk about true scores. Instead, we refer to the systematic component of the forecast. The error component is sometimes called the unsystematic component. This helps distinguish between the error that contributes to unreliability and forecast error in the usual sense, that is, the difference between what is forecast and what actually happens.

The systematic component of a forecast is that part of the forecast that is systematically related to the information that is available at the time of the forecast. Given the same information, the systematic component will be the same; it is repeatable and therefore reliable. But there is an unsystematic component of the forecast that is unrelated in any way to the information that is available at the time of the forecast. It could be caused by forecaster inattention, distraction, indigestion, or any of a host of other factors. It is usually treated as random error and not analyzed. The variance of a set of forecasts is equal to the sum of the variances of the systematic and the unsystematic components. Whatever forecast accuracy is achieved is due to the systematic component (except of course, for short-term chance relations). Accuracy is reduced if there is any unsystematic component in the forecast. This is not to say that all forecast errors are due to the unsystematic component. The systematic component can also contribute to forecast error.

In principle, the systematic component could be estimated by averaging many forecasts made by the same person or group under identical conditions. For practical purposes, reliability is the extent to which the same forecasts are made on different occasions under very similar conditions. It would be estimated by the correlation $\left(r_{Y Y}\right)$ between two sets of forecasts made under conditions that are similar in all important respects. With some assumptions, it can be shown that

$$
r_{Y Y}=\frac{\sigma_{t}^{2}}{\sigma_{t}^{2}+\sigma_{e}^{2}}
$$

In other words, reliability is the ratio of systematic (true) variance $\left(\sigma_{t}^{2}\right)$ in the forecasts to that variance plus unsystematic (error) variance $\left(\sigma_{\mathrm{e}}{ }^{2}\right)$. Reliability can be perfect (1.0) only if there is no unsystematic variance in the forecast.

Reliability is necessary, but not sufficient, for validity (i.e., accuracy) of forecasts. It is easy to construct examples of forecasts that are perfectly reliable but are inaccurate and of no practical value. A forecaster who predicted a temperature equal to the day of the month every day (one degree on the first, two degrees on the second, and so forth) would produce very reliable forecasts, but they would have no validity. The lens model equation (see below) describes the relation between reliability and accuracy. 


\section{Measurement of Forecast Reliability}

The reliability of a forecast can be estimated using three different approaches: (1) repeated judgments, (2) regression models, and (3) agreement among forecasters.

\section{Repeated judgments}

Reliability is often measured in controlled studies by computing correlations between repeated judgments made under very similar conditions (e.g., Lee and Yates 1992). In many forecasting situations, conditions rarely repeat, so reliability has to be estimated in other ways.

\section{Regression models}

Forecasts are based on a number of variables, which I will call cues. If a representative sample of forecasts is available, and the cues that each forecast was based on are known, and the sample is large enough to produce reasonably stable results, then multiple regression analysis can be used to model the forecasts. This technique, known as judgment analysis (Cooksey 1996), has been used extensively in judgment research. Assuming that the regression model captures all of the reliable variance in the forecasts, the multiple correlation between the forecast and the cues $\left(R_{Y . X}\right)$ would be equal to the square root of the reliability of the forecasts. Consequently $R_{Y . X}^{2}$ (actually, adjusted $R_{Y . X}^{2}$, which is an unbiased estimator of the population value, is preferred) could be considered an indicator of reliability. In practice, however, $R_{Y . X}$ depends on both reliability and the ability of the regression model to capture the underlying judgment process. If the regression model is a poor model of judgment, then $R_{Y . X}$ could be low even though reliability was high. Fortunately, it appears that regression models provide good models of judgment in a variety of situations. Many studies have found that simple linear regression models accurately reproduce expert judgments (Camerer 1981; Dawes 1979; Dawes and Corrigan 1974; Goldberg 1968; Slovic and Lichtenstein 1971). As a result, multiple regression analysis of judgment often provides a reasonable indicator of reliability. Balzer, Rohrbaugh, and Murphy (1983) compared regression and repeated judgment estimates of reliability for a preference judgment (not forecasting) task. They mistakenly concluded that regression estimates were higher because they reported $R_{Y . X}$ instead of adjusted $R_{Y . X}^{2}$. When the adjusted $R_{Y . X}^{2}$ value is estimated from their results, the value is .79 , which is comparable to the average reliability of 72 obtained using repeated judgments. Ramanaiah and Goldberg (1977) report a correlation of .89 between reliability and the multiple correlation for 83 subjects, thus suggesting that the multiple correlation can be a reasonable indicator of reliability.

\section{Agreement among forecasters}

Agreement among forecasters, as measured by the correlation between their forecasts, is an indirect indicator of reliability. If the random errors in two forecasts are independent, then the expected value of the correlation between them cannot exceed the product of the square roots of their reliabilities (Guilford 1954). Sample values of the correlation between two forecasts will exceed the product of the square roots of their reliabilities only by chance. Therefore, the correlations among forecasts can be used to estimate a lower bound on reliability. Of course, differences in cue utilization across individuals will also depress 
the correlations among their forecasts. The extent to which this is a factor in determining the correlation between forecasters will depend on the nature of the task (Stewart, Roebber and Bosart 1997).

\section{The Lens Model and the Importance of Judgmental Reliability}

A useful framework for understanding the role of reliability in judgmental forecasting is Brunswik's lens model, which conveniently comes with a matching equation-the aptly named "lens model equation." This equation is handy for quantifying the effect of reliability, or unreliability, on forecasting accuracy.

In judgmental forecasting the forecaster makes a prediction about something that cannot be known directly (the future event being forecast). That prediction is based on multiple cues (i.e., the variables representing the information available at the time the forecast is made) that are (1) imperfectly related to the future event, and (2) correlated with one another. The term cue is used in the psychological literature to denote a variable, factor, or indicator that the forecaster uses.

This view of judgmental forecasting is represented in the lens model (Exhibit 1). The right side of the lens model represents the relations between the available cues $(X)$ and the judgmental forecast $(\mathrm{Y})$. The left side of the lens model represents the relations between the cues and the event that is being forecast $(\mathrm{O})$. The lines connecting the cues and the actual event represent the ecological validities of the cues, that is, how the cues are related to the forecast event in the environment. The term "environment" is used to refer to the forecasting environment, not necessarily to nature. Finally, the arc connecting the criterion and the judgment represents the accuracy of the forecast. From the standpoint of the forecaster, the environment is fundamentally probabilistic. Forecasts will never be perfectly accurate.

\section{Exhibit 1}

Brunswik's lens model

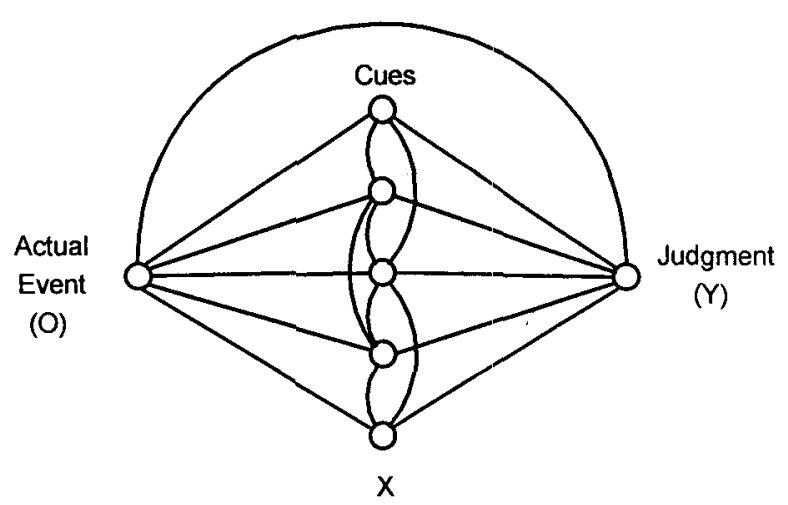

A special case of judgmental forecasting is the judgmental extrapolation of time series, which is discussed elsewhere in this volume (Harvey 2001; Webby, O'Connor, and Lawrence 2001). Conceptually, such forecasts can be represented in lens-model terms by considering the cues to be various features of the time series (trend, elevation, cycles, last 
point in the series, etc.). To my knowledge, no one has attempted to analyze judgmental extrapolation in lens-model terms.

The lens-model equation (LME) is a mathematical expression of fundamental concepts in Brunswik's $(1952 ; 1956)$ probabilistic functionalism and Hammond's social judgment theory (Hammond, et al. 1975). With some assumptions, it can be used to draw the following conclusions (see Appendix for details):

$$
\left(\begin{array}{c}
\text { Forecast } \\
\text { accuracy }
\end{array}\right) \approx\left(\begin{array}{c}
\text { Environmental } \\
\text { uncertainty }
\end{array}\right) \times\left(\begin{array}{c}
\text { Match between } \\
\text { forecast and } \\
\text { environment }
\end{array}\right) \times\left(\begin{array}{c}
\text { Forecast } \\
\text { reliability }
\end{array}\right)
$$

For our purposes, the most important thing to know about this equation is that all three terms on the right can be no greater than 1.0, and their product is a measure of forecast accuracy. Consequently judgmental unreliability places an upper bound on accuracy, and accuracy is reduced in proportion to the amount of reliability in judgment. This is important: The accuracy of a set of judgmental forecasts (1) can be no greater than its reliability and (2) is reduced in proportion to the amount of unreliability.

The other terms are important too. Environmental uncertainty is the predictability in the environment, given the available information. It places a ceiling on forecast accuracy that can be raised only by obtaining better information. The match between the forecast and the environment is the degree of similarity between the way the forecaster uses the cues and the way they should be used to maximize accuracy. In other words, it is a measure of the decrease in accuracy due to misuse of information. In this chapter I will focus on reliability.

\section{The Expanded Lens Model and the Components-of-skill Framework}

Stewart and Lusk (1994) derived an extension of the LME (see Appendix) based on an expanded lens model (Exhibit 2) that shows (1) that the cues available to the judge may be imperfect indicators of true descriptors, and (2) that the subjective cues that are integrated into a judgment may be imperfectly related to the objective cues. The important implication for us is that the expanded lens model indicates that reliability has two parts. One part is the reliability of acquiring information and the other is the reliability of processing information. In effect, the reliability of judgmental forecasts is the product of two kinds of reliability:

(1) reliability of information acquisition

(2) reliability of information processing.

Reliability of information acquisition is the relation between the objective cues available to the forecaster $(\mathrm{X})$ and the subjective cues that are integrated into the forecast (U). If, for example, a weather forecaster must use a radar display to judge the size of a storm to forecast severe weather, that judgment will not be perfectly reliable. This is unreliability of information acquisition, and it degrades the quality of the forecast. Empirical estimates of unreliability in information acquisition could be obtained by having forecasters make repeated cue judgments from the same data or by having different forecasters judge cues based on the same data. 
Exhibit 2

An expanded version of Brunswik's lens model

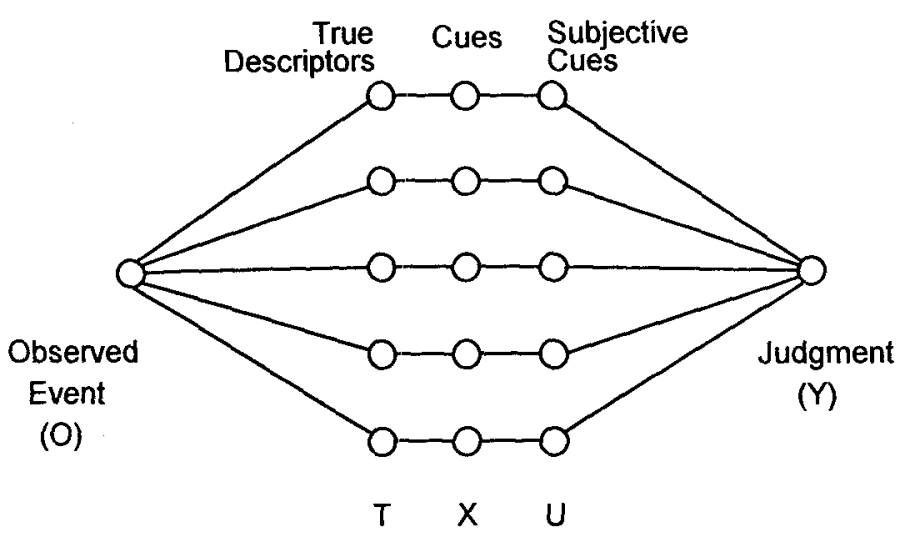

Reliability of information processing is the relation between the information acquired (subjective cues- $\mathrm{U}$ ) and the forecast $(\mathrm{Y})$. Reliability of information processing is less than perfect if, given the same subjective cues on two different occasions, forecasters produce different forecasts.

\section{Research on Reliability of Judgment}

Reliability has long been a concern in judgment research (e.g., Einhorn 1974; Slovic 1972; Slovic and Lichtenstein 1971), and research on the reliability of judgment has been reviewed recently by Stewart and Lusk (1994) and Harvey (1995).

Judgments are rarely perfectly reliable, and expertise does not appear to mitigate the problem of unreliability of judgment. Physicians (Kirwan et al. 1983; Levi 1989; Ullman and Doherty 1984), teachers (Brehmer and Brehmer 1988), clinical psychologists (Little 1961; Millimet and Greenberg 1973), neuropsychologists (Garb and Schramke 1996) grain inspectors (Trumbo et al. 1962) and weather forecasters (Lusk and Hammond 1991; Stewart, Moninger, Grassia et al. 1989) have been shown to be less than perfectly reliable judges.

Little is known about the relation between expertise and reliability. Although Bolger and Wright (1992) include "reliability" in the title of their chapter on expert judgment, they write primarily about validity. The only reliability studies they cite are a handful of multiple cue judgment studies showing a wide range of reliabilities for experts in different fields. In a recent review article on expert performance, Ericsson and Lehmann (1996) do not mention reliability at all. In several studies of weather forecasters, my colleagues and I have addressed reliability, but only indirectly. Although individual differences in reliability have been observed (e.g., Balzer, Rohrbaugh, and Murphy 1983) little is known about reasons for such differences. The LME clearly predicts a relation between reliability and accuracy, but no one has demonstrated empirically that experts that are more reliable are in fact more accurate.

Harvey (1995) identified six possible explanations for the lack of reliability of judgment: (1) failure of cognitive control (Hammond and Summers 1972), (2) overloading 
working memory, (3) recursive weight estimation during learning, (4) learning correlations rather than learning functions, (5) reproducing noise, and (6) deterministic rule switching. He reported an experiment involving extrapolation of time series in which people appeared to simulate the noise in the task. That is, if the historical time series was noisy, they made the forecast noisy, which is a poor strategy.

In laboratory studies, several potential task variables have been found to influence judgmental reliability. It is well-established that task predictability affects reliability. A number of researchers have found evidence that the reliability of judgment is lower for less predictable tasks (Brehmer 1976; 1978; Camerer 1981; Harvey 1995). This point deserves emphasis: Judges respond to unpredictable tasks by behaving less predictably themselves.

It has been suggested that judgments become less reliable as the amount of information available increases (Einhom 1971, Hogarth 1987). Although this seems to be widely accepted among judgment and decision researchers, it is an effect that rarely occurs to experts who do not have a background in statistics or psychometrics. Despite the acceptance of this relation, we have been able to find only one direct empirical test of it (Lee and Yates 1992). They found that increasing the number of cues from two to three resulted in no decline in reliability. In a study of forecasting from time series, Lawrence and O'Conner (1992) found that forecasters performed significantly worse when presented with larger time series ( 40 vs. 20 points). Wagenaar and Timmers (1978) reported a similar finding. Stewart et al. (1992) argued, based on indirect evidence, that decreased reliability was partially responsible for the lack of improvement in weather forecasts when increased amounts of information were provided. Although additional information could serve to improve the forecaster's understanding of the environmental conditions at the time of the forecast, it also increases the complexity of the forecasting task and may impose a cognitive burden on the forecaster that exceeds human information processing capacity.

Some indirect evidence regarding the relation between amount of information and reliability of judgment comes from studies showing that greater task complexity is associated with less reliability in judgments (Brehmer and Brehmer 1988). As more information becomes available, the complexity of the task increases (Einhorn 1971, Sen and Boe 1991). Faust (1986) reviewed several studies suggesting that judges are not able to make proper use of large numbers of cues. It is not surprising, therefore, that a number of studies have found that people use only a subset of available information (Brehmer and Brehmer 1988) and that the accuracy of forecasts does not increase as information increases (Armstrong 1985, Brockhoff 1984, Lusk and Hammond 1991).

A number of factors that might affect reliability have not been studied or have received almost no attention. For example, surprisingly, the effect of stress on judgmental reliability has not been studied. One researcher (Rothstein 1986) found that reliability decreased with increasing time pressure, which is a stressor. Another example is the relation between reliability and certainty of judgment. Little (1961) found a relation between certainty and reliability of the judgments of clinical psychologists. When the psychologists indicated that they were more certain about a judgment, they were also more reliable. Since subjective certainty (or confidence) is related to task predictability, task complexity, and amount of information, this result is potentially important, but further investigation of the relation is needed. 


\section{Research on Reliability of Information Acquisition}

Reliability of information acquisition is the extent to which the forecaster can reliably make use of available information and displays to infer subjective cues from the objective cues. The evidence suggests that unreliability of information acquisition is pervasive. It is more likely to be a problem in tasks, such as weather forecasting or medical diagnosis, which require interpretation of images or recognition of complex patterns in data that are distributed over time or space. In a review of research on reliability of clinical judgments in medicine, Koran (1975) found a wide range of intra- and interobserver reliability in extracting cardiovascular, gastrointestinal and respiratory cues from physical examination. He further reports a range of reliabilities for interpreting diagnostic procedures (e.g., electrocardiography). Einhorn (1974) studied pathologists viewing biopsy slides of cancer patients and reports a wide range of mean intrajudge reliabilities for cues. We have compiled a bibliography of recent medical studies that measured reliability of information acquisition (Stewart, Bobeck and Shim 2000). Reliabilities range from very low (e.g., determining whether a patient is wheezing) to quite high (e.g., determining whether a patient smokes).

Results of an experiment conducted by Brehmer (1970) indicated that unreliability in judging cues made learning more difficult and had an effect similar to that of unpredictability in the environment. This suggests that unreliability in information acquisition may affect not only the quality of the forecasts but also forecasters' ability to learn from experience.

Lusk and Hammond (1991) distinguish between primary cues that are directly observable from the presented information and secondary cues that must be extracted or inferred from a combination of the primary-cue values. In studies comparing presentation of primary and secondary cues, they found more disagreement among weather forecasters' probability judgments in the primary cue condition than in the secondary cue condition, which they suggest was due to differential integration of the primary cue information into secondary cue values. They also found that the degree of disagreement on secondary cue values varied considerably by cue. They suggest that this may have been related to differences in the proximity of the secondary cues to the primary cues. That is, the differences may be due to the varying degrees of subjectivity involved in making the secondary cue judgments.

A special case of secondary cues are cues that describe future, rather than current, conditions and therefore must themselves be forecast. The evidence reviewed by Armstrong, Brodie, and McIntyre (1987) indicates that unreliability introduced by integrating information to forecast a cue may not be a serious problem. They reviewed 18 studies comparing conditional econometric forecasts (actual data on the causal variables) and unconditional forecasts (causal variables must be forecast) and found that 10 studies showed that conditional forecasts were less accurate than unconditional forecasts, five showed no difference, and only three studies showed greater accuracy for conditional forecasts.

Despite its importance, we have not found any studies that specifically evaluate methods for improving reliability of information acquisition in forecasting. There are, however, several general suggestions that deserve study. Lusk et al. (1990) recommend that clear operational definitions be developed for each cue. Lusk and Hammond (1991) suggest that identification of specific cues demonstrating high levels of disagreement among forecasters would make it possible to focus on variables with the greatest potential for improving 
judgment. Reliability might also be improved through forecaster training focused on troublesome cues or by designing improved displays, taking into account factors that affect reliability.

\section{Intuition and Analysis Produce Different Kinds of Error}

Judgments involved in forecasting involve both analytic and intuitive processes, just as all judgments do. Hammond (1996) provides a compelling discussion of analysis and intuition, and the strengths and limitations of each. He begins:

The meaning of analysis or analytical thought in ordinary language is clear; it signifies a step-by-step, conscious, logically defensible process. The ordinary meaning of intuition signifies the opposite-a cognitive process that somehow produces an answer, solution, or idea without the use of a conscious, logically defensible, step-by-step process. Analysis has always had the advantage over intuition with respect to the clarity of its definition for two reasons: (1) its meaning could be explicated by the overt reference to a logical and/or mathematical argument, and (2) analytical thought forms the basis of rationality, because rational argument calls for an overt, step-by-step defensible process. Thus, analytical thought and explicit, overt definition are part of the same system of thought. Not so with intuition; throughout history it has acquired powerful claims to efficacy despite its ineffable, undefinable character (p. 60).

Following Brunswik, Hammond argues that judgment is quasi-rational, that is, it involves elements of both analysis and intuition. He further argues that intuition and analysis define a continuum, rather than a dichotomy, and that cognitive processes involved in a particular judgment task are located at a point on that continuum determined by properties of the task and the judge.

For the purposes of this chapter, the important difference between intuition and analysis is that they produce different kinds of errors, and that leads to different conclusions about reliability.

Brunswik demonstrated the difference between the errors of analytic and intuitive cognition as follows.

He asked subjects to estimate the height of a bar intuitively (by eye, that is) and then examined the distribution of errors. The error distribution followed the normal (bell-shaped) curve, with the mean judgment at approximately the right answer (Exhibit 3a). He then asked a second group to calculate the height of the bar by means of trigonometry. Most of the calculated answers were exactly correct, but those that weren't were far off the mark [Exhibit $3 b$ ]. Intuitive perception is robust but imprecise; analytical cognition is precise, but subject to large error-when errors are made. (See Hammond 1996, p. 160.). Peters et al. (1974) obtained similar results, as did Hammond, et al. (1987).

This means that both intuitive and analytic processes can be unreliable, but different kinds of errors will produce that unreliability. If we represent reliability graphically as a scatterplot of the relation between repeated judgments, an intuitive process would appear 
as the familiar elliptical pattern produced by the bivariate normal distribution (Exhibit $3 \mathrm{c}$ ). The plot for an analytic process would look like a straight line with a few extreme outliers (Exhibit $3 \mathrm{~d}$ ). For both plots, the correlation is approximately .7, but the process that produces that correlation and the implications for forecast accuracy are quite different.

It is generally assumed that analytic processes are more reliable than intuitive processes. For example, a computer-forecasting model is an analytic process that will always produce the same results given the same inputs. The reliability of such models is the primary reason that statistical models often outperform human judges (Dawes and Corrigan 1974, Grove and Meehl 1996) and that models of judges often outperform the judges themselves (i.e., judgmental bootstrapping. See Armstrong 2001, Camerer 1981, Goldberg 1970).

In practice, however, analytic processes are not perfectly reliable. Small errors in inputs can produce large output errors. System failure (O'Connor, Doherty, and Tweney 1989) can also produce large errors. When errors are produced, they can be catastrophic (see Hammond, 1996, for numerous examples).

Exhibit 3

Schematic comparison of error distribution and reliabilities of intuitive and analytic forecasting processes

3a. Distribution of errors for intuitive process

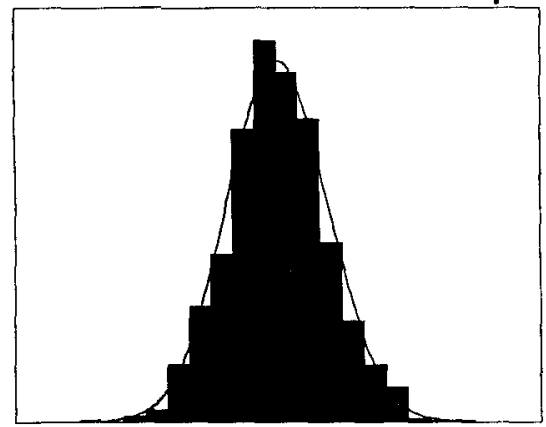

Intuitive forecast

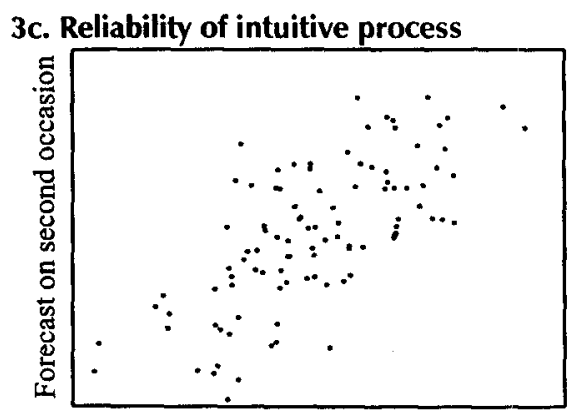

Forecast on first occasion 3b. Distribution of errors for analytic process

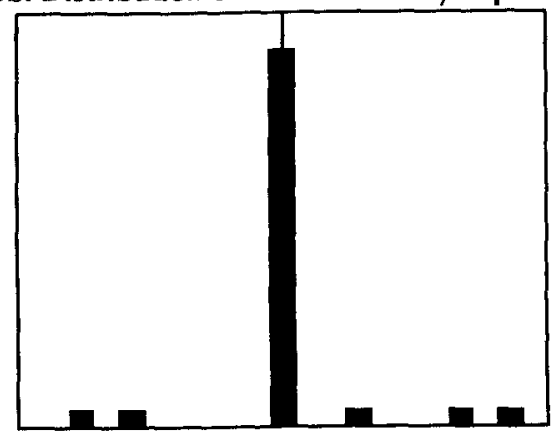

Analytic forecast

3d. Reliability of analytic process

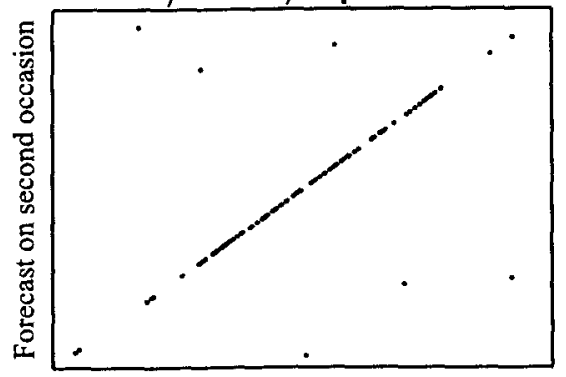

Forecast on first occasion 


\section{SUMMARY}

Does reliability affect judgmental forecasts? The simple answer is yes. People are not perfectly reliable, and that contributes to forecasting errors. Furthermore, there is evidence that simple techniques for increasing reliability, such as bootstrapping and averaging multiple forecasts, improve accuracy.

The answer to the important follow-up question is not so simple: How much does reliability affect judgmental forecasts? It depends on the forecasting problem, the forecaster, and the method used. I make the following generalizations from the research:

Generalization: Reliability decreases as task predictability decreases.

Implication: Reliability is a greater problem when a highly uncertain event is being forecast.

Generalization: Reliability decreases as task complexity (e.g., amount of information) increases.

Implication: Increasing the amount of information available to the forecaster may not improve the quality of the forecast.

Generalization: Forecasting processes that are highly intuitive will generally result in less reliable forecasts than those that are highly analytic (although analytic processes are rarely perfectly reliable).

Implication: Since all forecasts require a combination of analysis and intuition, the role of each process should be carefully considered and the forecasting process should be structured to take advantage of the strengths of both processes while avoiding their limitations.

Generalization: Reliability of information acquisition will be lower for tasks that involve perception (e.g., pattern recognition) or judgmental interpretation in the acquisition of information.

Implication: For such tasks, improvements in information displays are likely to produce gains in accuracy.

\section{PRINCIPLES}

Five principles for improving reliability can be put forward with some confidence. Only one of these directly addresses information acquisition; the other four address information processing.

\section{Addressing the Problem of Reliability of Information Acquisition}

One principle applies to reliability of information acquisition, but it is based more on theory and common sense than on an empirical body of research.

- Organize and present information in a form that clearly emphasizes relevant information. 
Specifically, use unambiguous information displays. Avoid displays that require recognition of complex patterns or mental aggregation of many numbers to obtain a cue. Avoid reliance on short-term memory. Make it easier to acquire relevant information reliably. Usually, paying attention to the most relevant information and ignoring irrelevant information is more important than how that information is processed. Highlight relevant information. Remove irrelevant information.

Purpose: To reduce errors due to unreliability in information acquisition.

Conditions: This principle should be applied whenever the cues are themselves judged or forecast or must be acquired perceptually. This problem arises in perception- and imageintensive activities such as weather forecasting, medical diagnosis, personnel selection, and legal proceedings involving eyewitness and expert testimony. It is not as important in business and economic forecasting situations where most of the data are numerical, unless the forecaster attempts to mentally analyze a set of numbers to detect a pattern that serves as a cue.

Evidence: The lens-model equation shows how errors in information acquisition reduce forecast accuracy. Perceptual processes, memory, and mental aggregation of data introduce errors.

In a metanalysis of 111 studies of judgments based on personnel selection interviews, Conway, Jako, and Goodman (1995) found clear evidence for the importance of structure in improving both the reliability and the validity of judgments. Specifically, they found a strong relation between standardization of questions and reliability of judgments. This finding is directly relevant to the proposed forecasting principle because question standardization facilitates the reliable acquisition of relevant information.

\section{Addressing the Problem of Reliability of Information Processing}

Research offers a sound basis for several principles that can improve the reliability of information processing.

\section{- Limit the amount of information used in judgmental forecasting. Use a small number of very important cues.}

When the number of cues cannot be limited, it may be possible to decompose a complex judgment task into a several simpler tasks (Edmundson 1990, MacGregor 2001). Reliability for each of the simpler tasks should be greater than for the complex task.

Purpose: To improve reliability of information processing and limit the errors introduced by overreliance on less relevant cues or distractions due to irrelevant cues.

Conditions: This principle applies any time several cues are processed judgmentally in forecasting, but it will be more important when large amounts of information are potentially available. The greatest benefit would be expected when environmental uncertainty is moderate to high and no analytic method for processing information is available. 
Evidence: As the number of cues increases, judgmental reliability decreases.

\section{- Use mechanical methods to process information.}

Mechanical method generally refers to a computerized model. The model need not be complex. Simple linear models will often be useful.

Purpose: To improve reliability of information processing by substituting an analytical process for an intuitive one. The systematic components of the analytic and intuitive processes should be closely matched, but the analytic process will have a smaller unsystematic (error) component.

Conditions: This principle can be used when information can be processed mechanically without losing important cues. Greater benefit can be expected from applying this principle when the forecasting environment contains a high degree of uncertainty.

Evidence: This principle is based on the superior reliability of analytic models. The LME shows why accuracy is increased when a more reliable processing system is used. To achieve this increased accuracy, however, the analytic model must have access to all of the important information available for the forecast. It is not necessary to use a complex model for processing information. A large body of research suggests that when people and analytic models have access to the same information simple linear models produce results that are at least as accurate as the humans and often more accurate (Grove and Meehl 1996). Research on judgmental bootstrapping (which preceded and is not related to statistical bootstrapping) looked at what happens when a regression model of judgment is substituted for the original judgments. Often the perfectly reliable regression model performs better than the original judgments used to derive it (Armstrong 2001, Camerer 1981, Goldberg 1970). Cooksey, Freebody, and Bennett (1990) and Ramanaiah and Goldberg (1977) found that the reliability of bootstrapped judgments was higher than the reliability of the judgments themselves.

Although it cannot be implemented in every forecasting or judgment situation, this principle has as much solid support from judgment and decision research and theory as any recommendation that could be made. At least as early as 1972, researchers suggested that expert judgment could be improved by having experts judge the cues, where necessary, and then use models to process the information (Einhorn 1972). Einhorn suggested that humans are better at information acquisition while machines are better at information processing. Despite the long history of this idea, it is regularly rediscovered, often with great fanfare. For example, a recent article on the first page of the business section of the New York Times touted a "revolutionary new way to approve small business loans" (Hansell 1995). The article claimed that the new method would save time and cut the number of bad loans. The "revolutionary" method was based on use of a simple computerized model to process loan applications. A similar recommendation, based on empirical evidence, had been made 20 years earlier by Wilsted, Hendrick and Stewart (1975).

It is important to remember that nearly all of the research demonstrating the superiority of mechanical information processing assumes that models and humans have access to exactly the same information. This is unlikely to be true in real forecasting situations. When humans and models have access to different information, humans can be more accurate than the models, and, more importantly, some combination of models and machines 
might be more accurate than either (Blattberg and Hoch 1990; Murphy and Brown 1984; Roebber and Bosart 1996).

Based on their metanalysis of 111 personnel selection judgment studies, Conway, Jako and Goodman (1995) strongly recommend mechanical combination of ratings of characteristics of the person interviewed. Their recommendation carries substantial weight because it is not based on logical arguments regarding the reliability of models versus humans, but rather on empirical comparisons of actual results based on subjective and mechanical combination.

While use of a model for processing information virtually guarantees increased reliability (at the same time, as discussed above, introducing the possibility of an occasional catastrophic error), that does not necessarily mean that models should be used in every situation and certainly does not imply that judgment should be excluded from forecasting, even if that were possible. For a more complete discussion of the issues involved in the use of models in forecasting, see Bunn and Wright (1991).

\section{- Combine several forecasts.}

Purpose: To improve reliability of information processing.

Conditions: It is possible to obtain more than one independent judgmental forecast.

Evidence: Combining forecasts by mathematically aggregating a number of individual forecasts increases the reliability of forecasts (Kelley 1925, Stroop 1932) and averages out unsystematic errors (but not systematic biases) in cue utilization. A common method for combining individual forecasts is to calculate an equal weighted average of the individual forecasts.

Research on group judgment has long shown that the mathematical aggregation of judgments from several individuals (or the aggregation of several judgments from one individual) tends to be more accurate than would be expected by randomly selecting a single individual from the population of all prospective group members (Bruce 1935, Gordon 1924, Stroop 1932). Furthermore, studies of weather forecasting (Bosart 1975, Sanders 1963), sales forecasting (Ashton and Ashton 1985), and economic forecasting (McNees 1987) suggest that group average forecasts based upon equal-weighted models tend to be more accurate than most individual forecasts.

The conditions under which combining forecasts is most likely to increase accuracy have been thoroughly analyzed (Clemen 1989, Maines 1990, Winkler and Makridakis 1983). The accuracy of a mathematically aggregated forecast is a function of the accuracy of the individual forecasts and the correlations among their errors (Maines 1990). For best results, the forecasts to be combined should, to the extent possible, be based on different assumptions or independent information (Bunn 1987, Winkler 1981), but the information that goes into each forecast should also be significantly related to the event being predicted (McNees 1987). It is important that the correlations among forecast errors be as low as possible (Bunn 1987, Maines 1990). Consequently, aggregation of forecasts is likely to be most successful when the accuracy of each forecast is maximized while the intercorrelations among them are minimized. Under these conditions, aggregation of forecasts can enhance accuracy because the unsystematic variance in the individual forecasts will tend to cancel out and the valid systematic variance will be emphasized.

Because larger sample sizes produce more reliable averages, judgmental accuracy should increase with the size of the group of judges aggregated. Both theoretical and em- 
pirical work on the aggregation of judgment suggests that much of the gain in forecast accuracy that can be achieved through aggregation can be realized by combining a small number of forecasts (Ashton and Ashton 1985, Ashton 1986; Einhorn, Hogarth and Klempner 1977; Makridakis and Winkler 1983). The number of experts that should be included in an aggregate forecast is dependent on the amount of systematic bias in the forecasts (Einhorn, Hogarth and Klempner 1977). However, if there is a great deal of systematic bias in prediction, the accuracy of aggregated group judgment may be worse than the accuracy expected by randomly selecting a single individual from the population of all prospective group members (Preston 1938, Smith 1941). See also Gigone and Hastie (1997) for an excellent review of research on the accuracy of group judgment.

One should be aware of two important cautions when combining forecasts. First, aggregation will not always increase the accuracy of forecasts. If forecasts with zero accuracy are combined, the result will have zero accuracy. If forecasts with negative accuracy are combined, the results will have even greater negative accuracy (which, of course, can be useful if the user knows enough to reverse the forecast before using it). If forecasts with negative and positive accuracy are combined, the result may have zero accuracy.

Second, by simply averaging the forecasts of experts who disagree, the practitioner may overlook an opportunity to improve forecasts by determining why experts disagree and using that knowledge to develop a better forecast (Stewart 1987). If forecasts disagree greatly due to systematic differences between experts, rather than just due to unreliability, it is better to implement a process designed to understand and resolve the source of the disagreement (e.g., Hammond, Anderson, Sutherland, and Marvin 1984) rather than simply averaging disparate forecasts, which amounts to sweeping the disagreement under the rug.

Some forecasters may be systematically optimistic or pessimistic in their forecasts because they are concerned about different effects of overforecasting and underforecasting. That is, they have an asymmetric loss function. For example, state revenue forecasters who work for legislators of one party might overestimate revenues to justify greater spending. At the same time, revenue forecasters working for a governor of another party might underestimate revenues to trigger spending cuts. Although an ideal forecast would be free of values and not influenced by any considerations other than accuracy, real forecasts are frequently biased to serve the interests of the forecasters or their employers.

How do you determine whether disagreement among forecasters is due to systematic or unsystematic variance? This generally requires a formal study. If the cues can be measured, then the lens model equation provides a method for analyzing disagreement into systematic and unsystematic components (for an example, see Stewart, et al. 1989).

\section{- Require justification of forecasts.}

Purpose: To improve reliability of information processing.

Conditions: This principle is likely to be most useful for tasks with low predictability because reliability of information processing is a more significant problem for such tasks.

Evidence: Hagafors and Brehmer (1983) suggest that reliability might increase if the forecaster were asked to justify forecasts verbally. They found that having to justify one's opinion led to higher consistency when no outcome feedback is provided. The effect of justification was higher in low predictability conditions than in high predictability condi- 
tions, suggesting an interaction between the benefits of justification and environmental predictability. They also found that outcome feedback reduced consistency. They suggest that subjects use feedback to test hypotheses, and the hypotheses keep changing, resulting in decreased reliability. Without feedback, hypothesis testing cannot occur and reliability increases. York, Doherty, and Kamouri (1987), however, found that outcome feedback does not always reduce reliability. It may be that outcome feedback can provide increased motivation that increases reliability (Annett 1969).

Requiring justification of forecasts will also move the forecasting process away from an intuitive process and toward an analytic process (Hammond 1996), and this can be expected to increase reliability.

\section{IMPLICATIONS FOR PRACTITIONERS}

In summary, practitioners should be aware that judgment has both positive and negative effects on forecast accuracy. One of the negative effects is the inevitable introduction of unreliability into the forecast. Errors due to unreliability can be addressed directly if practitioners are aware of the problem and consider alternative methods for making judgmental forecasts.

Explicit attention to reliability of judgment carries the potential for improved forecast accuracy. By instituting changes in procedures for judgmental forecasting along the lines described above, forecast accuracy can be improved with currently available information and models. In many settings, this will prove to be an inexpensive modification compared to the alternatives of obtaining more information and better models. In other settings, it may be the only option available.

\section{IMPLICATIONS FOR RESEARCHERS}

Researchers should address the conditions that produce unreliability. All important business, economic, and environmental forecasts involve some elements of uncertainty and complexity and require human judgment. As a result, their accuracy is reduced by judgmental unreliability. Little is known about the causes of unreliability or how much accuracy is lost due to unreliability in specific situations. By understanding the nature of unreliability and its impact on accuracy, we can design and evaluate methods for training forecasters, organizing information, and structuring the forecasting task to improve accuracy.

\section{SUMMARY}

The forecasting principles derived from theory and research on the reliability of judgment are these:

- Organize and present information in a form that clearly emphasizes relevant information. 
- Limit the amount of information used in judgmental forecasting. Use a small number of very important cues.

- Use mechanical methods to process information.

- Combine several forecasts.

- Require justification of forecasts.

Unreliability is inevitable in judgmental forecasting, and it reduces the accuracy of forecasts. We do not know enough about the size of the effect of unreliability on accuracy, or its causes or about how to improve reliability. We do know that it is rarely addressed explicitly in judgmental forecasts and that there are methods for addressing it that can be implemented at relatively low cost.

\section{APPENDIX: LENS MODEL EQUATION FORMULAS}

\section{The Lens Model Equation}

Hammond, Hursch and Todd (1964) and Hursch, Hammond and Hursch (1964) presented the original lens model equation, but Tucker (1964) proposed the form most used today. The LME decomposes the correlation ( $\mathrm{r}_{\mathrm{YO}}$ ) between a judgment $(\mathrm{Y})$ and the actual event $(\mathrm{O})$ and is based on a partitioning of each variable into two components-one that is a function of the cues $(\mathrm{X})$ used to make the judgment and another that is unrelated to them. This partitioning can be written as

$$
\begin{aligned}
& \mathrm{O}=\mathrm{M}_{\mathrm{O} . \mathrm{X}}\left(\mathrm{X}_{1}, \mathrm{X}_{2}, \ldots \mathrm{X}_{\mathrm{n}}\right)+\mathrm{E}_{\mathrm{O} . \mathrm{X}} \\
& \mathrm{Y}=\mathrm{M}_{Y . X}\left(\mathrm{X}_{1}, \mathrm{X}_{2}, \ldots X_{\mathrm{n}}\right)+\mathrm{E}_{\mathrm{Y} . \mathrm{X}}
\end{aligned}
$$

where $M_{O . X}$ and $M_{Y . X}$ represent models that describe the relations between the cues and the criterion and the cues and the judgment, respectively; and the E's, which represent the residuals or "errors" of the models, are not related to the M's. In the original papers, the models were assumed to have been derived using multiple regression analysis, but that is not a necessary condition. The lens model equation holds as long as the E's and the M's are uncorrelated.

Note that the two components of $\mathrm{Y}$ correspond to the systematic and unsystematic components of the forecast.

This partitioning of the judgment and the criterion can be used to derive a partitioning of the correlation between them (Stewart 1976). Based on such a partitioning, Tucker (1964) developed the following form of the lens model equation:

$$
r_{Y O}=R_{O . X} G_{Y . X}+C \sqrt{1-R_{O . X}^{2}} \sqrt{1-R_{Y . X}^{2}}
$$

where $\quad R_{O . X}$ is the correlation between $O$ and $M_{O . X}$,

$G$ is the correlation between $M_{O . X}$ and $M_{Y . X}$,

$R_{Y . X}$ is the correlation between $Y$ and $M_{Y . X}$, and

$\mathrm{C}$ is the correlation between $\mathrm{E}_{\mathrm{Y} . \mathrm{X}}$ and $\mathrm{E}_{\mathrm{O} . \mathrm{X}}$. 
If $M_{Y . X}$ is an adequate model of the judgments, then $R_{Y . X}$ can be considered an estimate of the reliability of judgment. Since the second term of the LME is generally small enough to be ignored, the equation can be simplified as follows:

$$
\mathrm{R}_{\mathrm{YL}} \cong \mathrm{R}_{\mathrm{O} . \mathrm{X}} \mathrm{GR}_{\mathrm{Y} . \mathrm{X}}
$$

Since all three terms on the right can be no greater than 1.0 , and their product is a measure of forecast accuracy ( $\mathrm{r}_{\mathrm{YO}}$ ), judgmental reliability places an upper bound on accuracy, and accuracy is reduced in proportion to the amount of reliability in judgment.

There have been a number of important methodological developments since the original 1964 papers. Castellan (1972) generalized the lens model to multiple criteria. Stenson (1974) showed how $\mathrm{G}$ could be estimated from the environmental and subject reliabilities if the cues were unknown, demonstrating the relation between $G$ and correction for attenuation of a validity coefficient in test theory. Stewart (1976) developed a hierarchical formulation that made it possible to isolate the contributions of different sets of variables. Cooksey and Freebody (1985) developed a fully generalized lens model equation that encompassed both the Castellan multivariate and the Stewart hierarchical formulations. Castellan (1992) explored the properties of $G$ under a variety of assumptions. Stewart (1990) combined the LME with a decomposition of the Brier skill score, incorporating regression and base-rate bias into the formulation. Based on an expanded version of the lens model, Stewart and Lusk (1994) decomposed $R_{0 . x}$ into environmental predictability and fidelity of the information system and $R_{Y, X}$ into reliability of information acquisition and reliability of information processing. For a more complete treatment of the lens model and the LME, see Cooksey (1996).

\section{The Expanded Lens Model Equation}

The expanded LME, based on Tucker (1964), Murphy (1988), and Stewart (1990), incorporates forecast bias by using a measure of accuracy based on the mean squared error and decomposes both environmental predictability and forecast reliability into two terms:

$$
\mathrm{SS}=\left(\mathrm{GR}_{\mathrm{O} . \mathrm{T}} \mathrm{V}_{\mathrm{T} . \mathrm{X}} \mathrm{R}_{\mathrm{Y} . \mathrm{U}} \mathrm{V}_{\mathrm{U} . X}\right)^{2}-\left[\mathrm{r}_{\mathrm{YO}}-\left(\frac{\mathrm{S}_{\mathrm{Y}}}{\mathrm{S}_{\mathrm{O}}}\right)\right]^{2}-\left[\frac{(\overline{\mathrm{Y}}-\overline{\mathrm{O}})}{\mathrm{s}_{\mathrm{O}}}\right]^{2}
$$

where $\mathrm{SS}$ is the skill score: $\mathrm{SS}=1-\left(\frac{\mathrm{MSE}_{\mathrm{Y}}}{\mathrm{MSE}_{\mathrm{B}}}\right)$;

$\mathrm{MSE}_{\mathrm{Y}}$ is the mean square error for the judgment, and $\mathrm{MSE}_{\mathrm{B}}$ is the mean square error for the base rate.

$\mathrm{G}$ measures the match between the environmental model and the judgment model (this is the traditional G from the Tucker 1964 LME);

$\mathrm{R}_{\mathrm{O} . \mathrm{T}}$ is a measure of the predictability of the environment, given true cues; 
$\mathrm{V}_{\mathrm{T} . \mathrm{X}}$ is a measure of the fidelity of the information system, that is, the reduction of skill due to degradation of the quality of information before it reaches the judge; $\mathrm{R}_{\mathrm{Y} . \mathrm{U}}$ is a measure of the forecaster's information processing reliability; $\mathrm{V}_{\mathrm{U} . \mathrm{X}}$ is a measure of the reliability of information acquisition.

$$
\left[\mathrm{r}_{\mathrm{YO}}-\left(\frac{\mathrm{s}_{\mathrm{Y}}}{\mathrm{s}_{\mathrm{O}}}\right)\right]^{2}
$$

is conditional bias (Murphy 1988), which is similar to regression bias (Dawes 1988, Hogarth 1987) in the judgment literature.

$$
\left[\frac{(\bar{Y}-\bar{O})}{s_{o}}\right]^{2}
$$

is unconditional bias (Murphy 1988), which is similar to base-rate bias (Bar-Hillel 1990 Lichtenstein, Fischhoff and Phillips 1982) in the judgment literature.

Stewart and Lusk (1994) show how the components of skill framework can be used to organize the literature on aids to judgment. They argue that, in important fields of professional judgment, such as medical diagnosis and weather forecasting, extensive effort and resources are applied to improving the fidelity of the information system (through improved instrumentation) and the predictability of the environment (by studies designed to gain a better understanding of environmental processes and by providing better information about the environment). However, little attention has been paid to the reliability of judgment.

\section{REFERENCES}

Annett, J. (1969), Feedback and Human Behaviour: The Effects of Knowledge of Results, Incentives, and Reinforcement on Learning and Performance. Baltimore: Penguin Books.

Armstrong, J. S. (1985), Long-range Forecasting: From Crystal Ball To Computer. (Second Edition ed.). New York: Wiley. Full text at hops.wharton.upenn.edu/forecast.

Armstrong, J. S. (2001), “Judgmental bootstrapping: Inferring experts' rules for forecasting." In J. S. Armstrong (ed.), Principles of Forecasting. Norwell, MA: Kluwer Academic Publishers.

Armstrong, J. S., R. J. Brodie \& S. H. McIntyre (1987), "Forecasting methods for marketing: Review of empirical research," International Journal of Forecasting, 3, 355-376. Ashton, A. H. \& R. H. Ashton (1985), "Aggregating subjective forecasts: Some empirical results," Management Science, 31, 1499-1508.

Ashton, R. H. (1986), "Combining the judgments of experts: How many and which ones?" Organizational Behavior and Human Decision Processes, 38, 405-414. 
Balzer, W. K., J. Rohrbaugh \& K. R. Murphy (1983), "Reliability of actual and predicted judgments across time," Organizational Behavior and Human Performance, 32, 109123.

Bar-Hillel, M. (1990), "Back to base rates," in R. M. Hogarth (ed.), Insights In Decision Making: A Tribute To Hillel J. Einhorn. Chicago: University of Chicago Press.

Blattberg, R. C. \& S. J. Hoch (1990), "Database models and managerial intuition: 50\% model + 50\% manager," Management Science, 36, 887-899.

Bolger, F. \& G. Wright (1992), "Reliability and validity in expert judgment," in F. Bolger $\&$ G. Wright (eds.), Expertise and Decision Support. New York: Plenum Press. (pp. $47-76)$

Bosart, L. F. (1975), "SUNYA experimental results in forecasting daily temperature and precipitation," Monthly Weather Review, 103, 1013-1020.

Brehmer, A. \& B. Brehmer (1988), "What have we learned about human judgment from thirty years of policy capturing?" in B. Brehmer \& C. R. B. Joyce (eds.), Human Judgment: The Social Judgment Theory View. Amsterdam: North-Holland. (pp. 75114).

Brehmer, B. (1970), "Inference behavior in a situation where the cues are not reliably perceived," Organizational Behavior and Human Performance, 5, 330-347.

Brehmer, B. (1976), "Note on the relation between clinical judgment and the formal characteristics of clinical tasks," Psychological Bulletin, 83, 778-782.

Brehmer, B. (1978), "Response consistency in probabilistic inference tasks," Organizational Behavior and Human Performance, 22, 103-115.

Brockhoff, K. (1984), "Forecasting quality and information," Journal of Forecasting, 3, $417-428$.

Bruce, R. S. (1935), "Group judgments in the fields of lifted weights and visual discrimination," Journal of Psychology, 1, 117-121.

Brunswik, E. (1952), The Conceptual Framework of Psychology. Chicago: University of Chicago Press.

Brunswik, E. (1956), Perception and the Representative Design of Psychological Experiments. 2nd ed. Berkeley: University of California Press.

Bunn, D. (1987), "Expert use of forecasts: Bootstrapping and linear models," in G. Wright \& P. Ayton (eds.), Judgemental Forecasting. Chichester: Wiley.

Bunn, D. \& G. Wright (1991), "Interaction of judgmental and statistical forecasting methods: Issues and analysis," Management Science, 37(5), 501-518.

Camerer, C. (1981), "General conditions for the success of bootstrapping models," Organizational Behavior and Human Performance, 27, 411-422.

Castellan, N. J. Jr. (1972), "The analysis of multiple criteria in multiple-cue judgment tasks," Organizational Behavior and Human Performance, 8, 242-261.

Castellan, N. J. Jr. (1992), "Relations between linear models: Implications for the lens model," Organizational Behavior and Human Decision Processes, 51, 364-381.

Clemen, R. T. (1989), "Combining forecasts: A review and annotated bibliography," International Journal of Forecasting, 5, 559-583.

Conway, J. M., R. A. Jako \& D. F. Goodman (1995), “A meta-analysis of interrater and internal consistency reliability of selection interviews," Journal of Applied Psychol$o g y, 80,565-579$.

Cooksey, R. W. (1996), Judgment Analysis: Theory, Methods, and Applications. New York: Academic Press. 
Cooksey, R. W. \& P. Freebody (1985), "Generalized multivariate lens model analysis for complex human inference tasks," Organizational Behavior and Human Decision Processes, 35, 46-72.

Cooksey, R. W., P. Freebody \& A. J. Bennett. (1990), "The ecology of spelling: A lens model analysis of spelling errors and student judgments of spelling difficulty," Reading Psychology: An International Quarterly, 11, 293-322.

Dawes, R. M. (1979), "The robust beauty of improper linear models in decision making," American Psychologist, 34 (7), 571-582.

Dawes, R. M. (1988), Rational Choice in an Uncertain World. New York: Harcourt, Brace, Jovanovich.

Dawes, R. M. \& B. Corrigan (1974), "Linear models in decision making," Psychological Bulletin, 81(2), 95-106.

Edmundson, R. H. (1990), "Decomposition: A strategy for judgmental forecasting," Journal of Forecasting, $9(4), 305-314$.

Einhorn, H. J. (1971), "Use of nonlinear, noncompensatory models as a function of task and amount of information," Organizational Behavior and Human Performance, 6, 127.

Einhorn, H. J. (1972), "Expert measurement and mechanical combination," Organizational Behavior and Human Performance, 7, 86-106.

Einhorn, H. J. (1974), "Expert judgment: Some necessary conditions and an example," Journal of Applied P'sychology, 59, 562-571.

Einhorn, H. J., R. M. Hogarth \& E. Klempner (1977), "Quality of group judgment," Psychological Bulletin, 84, 158-172.

Ericsson, K. A. \& A. C. Lehmann (1996), "Expert and exceptional performance: Evidence of maximal adaptation to task constraints," Annual Review of Psychology, 47, 273305.

Faust, D. (1986), "Research on human judgment and its application to clinical practice," Professional Psychology: Research and Practice, 17 (5), 420-430.

Garb, H. N. \& C. J. Schramke (1996), "Judgment research and neuropsychological assessment: A narrative review and meta-analysis," Psychological Bulletin, 120, 140153.

Gigone, D. \& R. Hastie (1997), "Proper analysis of the accuracy of group judgments," Psychological Bulletin, 121, 149-167.

Goldberg, L. R. (1968), "Simple models or simple processes? Some research on clinical judgments," American Psychologist, 23, 483-496.

Goldberg, L. R. (1970), "Man versus model of man: A rationale, plus some evidence, for a method of improving on clinical inferences," Psychological Bulletin, 73, 422-432.

Gordon, K. (1924), "Group judgments in the field of lifted weights," Journal of Experimental Psychology, 7, 398-400.

Grove, W. M. \& P. E. Meehl (1996), "Comparative efficiency of formal (mechanical, algorithmic) and informal (subjective, impressionistic) prediction procedures: The clinical/statistical controversy," Psychology, Public Policy, and Law, 2, 293-323.

Guilford, J. P. (1954), Psychometric Methods. (2nd ed.). New York: McGraw-Hill.

Hagafors, R. \& B. Brehmer (1983), "Does having to justify one's judgments change the nature of the judgment process?" Organizational Behavior and Human Performance, $31,223-232$. 
Hammond, K. R. (1996), Human Judgment and Social Policy: Irreducible Uncertainty, Inevitable Error, Unavoidable Injustice. New York: Oxford University Press.

Hammond, K. R., B. F. Anderson, J. Sutherland \& B. Marvin (1984), "Improving scientists' judgments of risk," Risk Analysis, 4, 69-78.

Hammond, K. R., R. M. Hamm, J. Grassia \& T. Pearson (1987), "Direct comparison of the efficacy of intuitive and analytical cognition in expert judgment," IEEE Transactions on Systems, Man, and Cybernetics, SMC-17, 753-770.

Hammond, K. R., C. J. Hursch \& F. J. Todd (1964), "Analyzing the components of clinical inference," Psychological Review, 71, 438-456.

Hammond, K. R., T. R. Stewart, B. Brehmer \& D. O. Steinmann (1975), “Social judgment theory," in M. F. Kaplan \& S. Schwartz (eds.), Human Judgment and Decision Processes. New York: Academic Press. (pp. 271-307).

Hammond, K. R. \& D. A. Summers (1972), "Cognitive control," Psychological Review, $79,58-67$.

Hansell, S. (1995) "Loans granted by the megabyte: Computer models change smallbusiness lending," The New York Times, (April 18), pp. D1, D4.

Harvey, N. (1995), "Why are judgments less consistent in less predictable task situations?" Organizational Behavior and Human Decision Processes, 63, 247-263.

Harvey, N. (2001), "Improving judgmental forecast," in J. S. Armstrong (ed.), Principles of Forecasting. Norwell, MA: Kluwer Academic Publishers.

Hogarth, R. (1987), Judgement and Choice (2nd ed.). New York: John Wiley \& Sons. Hursch, C. J., K. R. Hammond \& J. L. Hursch (1964), "Some methodological considerations in multiple-cue probability studies," Psychological Review, 71, 42-60.

Kelley, T. L. (1925), "The applicability of the Spearman-Brown formula for the measurement of reliability," Journal of Educational Psychology, 16, 300-303.

Kirwan, J. R., D. M. Chaput De Saintonge, C. R. B. Joyce \& H. L. F. Currey (1983), "Clinical judgment in rheumatoid arthritis," Annals of the Rheumatic Diseases, 42, 644-664.

Koran, L. M. (1975), "The reliability of clinical methods, data, and judgments," New England Journal of Medicine, 293, 642-646, 695-701.

Lawrence, M. \& M. O. Connor (1992), "Exploring judgmental forecasting," International Journal of Forecasting, 8, 15-26.

Lee, J W. \& J. F. Yates. (1992), "How quantity judgment changes as the number of cues increases: An analytical framework and review," Psychological Bulletin, 112 (2), 363377.

Levi, K. (1989), "Expert systems should be more accurate than human experts: Evaluation procedures from human judgment and decision making," IEEE Transactions on Systems, Man, and Cybernetics, 19 (3), 647-657.

Lichtenstein, S., B. Fischhoff \& L. D. Phillips (1982), "Calibration of probabilities: The state of the art to 1980," in D. Kahneman, P. Slovic \& A. Tversky (eds.), Judgment Under Uncertainty: Heuristics and Biases. New York: Cambridge University Press, pp. 306-334.

Little, K. B. (1961), "Confidence and reliability," Educational and Psychological Measurement, 21, 95-101.

Lusk, C. M. \& K. R. Hammond (1991), "Judgment in a dynamic task: Microburst forecasting," Journal of Behavioral Decision Making, 4, 55-73. 
Lusk, C. M., T. R. Stewart, K. R. Hammond \& R. J. Potts (1990), "Judgment and decision making in dynamic tasks: The case of forecasting the microburst," Weather and Forecasting, 5, 627-639.

MacGregor, D. G. (2001), "Decomposition for judgmental forecasting and estimation," in J. S. Armstrong (ed.), Principles of Forecasting. Norwell, MA: Kluwer Academic Publishers.

Maines, L. A. (1990), "The effect of forecast redundancy on judgments of a consensus forecast's expected accuracy," Journal of Accounting Research, 28, 29-47.

Makridakis, S. \& R. L. Winkler (1983), "Averages of forecasts: Some empirical results," Management Science, 29, 987-996.

McNees, S. K. (1987), "Consensus forecasts: Tyranny of the majority?" New England Economic Review (November/December), 15-21.

Millimet, C. R. \& R. P. Greenberg (1973), "Use of an analysis of variance technique for investigating the differential diagnosis of organic versus functional involvement of symptoms," Journal of Consulting and Clinical Psychology, 40, 188-195.

Murphy, A. H. (1988), "Skill scores based on the mean square error and their relationships to the correlation coefficient," Monthly Weather Review, 116, 2417-2424.

Murphy, A. H. \& B. G. Brown (1984), "A comparative evaluation of objective and subjective weather forecasts in the United States," Journal of Forecasting, 3, 369-393.

Nunnally, J. C. (1978), Psychometric Theory (2nd ed.). New York: McGraw-Hill.

O'Connor, R. M. Jr., M. E. Doherty \& R. D. Tweney (1989), "The effects of system failure error on predictions," Organizational Behavior and Human Decision Processes, $44,1-11$.

Peters, J. T., K. R. Hammond \& D. A. Summers (1974), "A note on intuitive vs analytic thinking," Organizational Behavior and Human Performance, 12, 125-131.

Preston, M. G. (1938), "Note on the reliability and validity of the group judgment," Journal of Experimental Psychology, 22, 462-471.

Ramanaiah, N. V. \& L. R. Goldberg (1977), "Stylistic components of human judgment: The generality of individual differences," Applied Psychological Measurement, 1, 2339.

Roebber, P. J. \& L. F. Bosart (1996), "The contributions of education and experience to forecast skill," Weather and Forecasting, 11, 21-40.

Rothstein, H. G. (1986), "The effects of time pressure on judgment in multiple cue probability learning." Organizational Behavior and Human Decision Processes, 37, 8392.

Sanders, F. (1963), "On subjective probability forecasting," Journal of Applied Meteorology, 2, 191-210.

Sen, T. \& W. J. Boe (1991), "Confidence and accuracy in judgements using computer displayed information," Behaviour \& Information Technology, 10, 53-64.

Slovic, P. (1972), "Psychological study of human judgment: Implications for investment decision making," Journal of Finance, 27(4), 779-799.

Slovic, P. \& S. Lichtenstein (1971), "Comparison of Bayesian and regression approaches to the study of information processing in judgment," Organizational Behavior and Human Performance, 6, 649-744.

Smith, B. B. (1941), "The validity and reliability of group judgments," Journal of Experimental Psychology, 29, 420-434. 
Stenson, H. H. (1974), "The lens model with unknown cue structure," Psychological Review, 81, 257-264.

Stewart, T. R. (1976), "Components of correlations and extensions of the lens model equation," Psychometrika, 41, 101-120.

Stewart, T. R. (1987), "The Delphi technique and judgmental forecasting," Climatic Change, 11, 97-113.

Stewart, T. R. (1990), "A decomposition of the correlation coefficient and its use in analyzing forecasting skill," Weather and Forecasting, 5, 661-666.

Stewart, T. R., J. Bobeck \& J. Shim (2000), Annotated bibliography and summary of medical literature on the reliability of diagnostic signs and judgments. Albany, NY: Center for Policy Research, Nelson A. Rockefeller College of Public Affairs and Policy, State University of New York.

Stewart, T. R. \& C. M. Lusk (1994), "Seven components of judgmental forecasting skill: Implications for research and the improvement of forecasts," Journal of Forecasting, 13, 579-599.

Stewart, T. R., W. R. Moninger J. Grassia, R. H. Brady \& F. H. Merrem (1989), "Analysis of expert judgment in a hail forecasting experiment," Weather and Forecasting, 4, 2434.

Stewart, T. R., W. R. Moninger, K. F. Heideman \& P. Reagan-Cirincione (1992), "Effects of improved information on the components of skill in weather forecasting," Organizational Behavior and Human Decision Processes, 53, 107-134.

Stewart, T. R., P. J. Roebber \& L. F. Bosart (1997), "The importance of the task in analyzing expert judgment," Organizational Behavior and Human Decision Processes, $69,205-219$.

Stroop, J. R. (1932), "Is the judgment of the group better than that of the average member of the group?" Journal of Experimental Psychology, 15, 550-560.

Trumbo, D., C. Adams, C. Milner \& L. Schipper (1962), "Reliability and accuracy in the inspection of hard red winter wheat," Cereal Science Today, 7, 62-71.

Tucker, L. R. (1964), "A suggested alternative formulation in the developments by Hursch, Hammond, and Hursch, and by Hammond, Hursch, and Todd," Psychological Review, $71,528-530$.

Ullman, D. G. \& M. E. Doherty (1984), "Two determinants of the diagnosis of hyperactivity: The child and the clinician," Advances in Developmental and Behavioral Pediatrics, 5, 167-219.

Wagenaar, W. A. \& H. Timmers (1978), "Extrapolation of exponential time series is not enhanced by having more data points," Perception and Psychophysics, 24, 182-184.

Webby, R., M. O'Connor \& M. Lawrence (2001), "Judgmental time-series forecasting using domain knowledge," in J. S. Armstrong (ed.), Principles of Forecasting. Norwell, MA: Kluwer Academic Publishers.

Wilsted, W. D., T. E. Hendrick \& T. R. Stewart (1975), "Judgment policy capturing for bank loan decisions: An approach to developing objective functions for goal programming models," Journal of Management Studies, 12, 210-215.

Winkler, R. L. (1981), "Combining probability distributions from dependent information sources," Management Science, 27, 479-488.

Winkler, R. L., \& S. Makridakis (1983), "The combination of forecasts," Journal of the Royal Statistical Society A, 146, 150-157. 
York, K. M., M. E. Doherty \& J. Kamouri (1987), "The influence of cue unreliability on judgment in a multiple cue probability learning task," Organizational Behavior and Human Decision Processes, 39, 303-317.

Acknowledgments: I gratefully acknowledge comments made by Nigel Harvey, Michael Doherty, Jeryl Mumpower, Ray Cooksey, Ken Hammond, and one anonymous reviewer on an earlier draft. 